\title{
Assessing the risk of measles resurgence in a highly vaccinated population: Belgium anno 2013
}

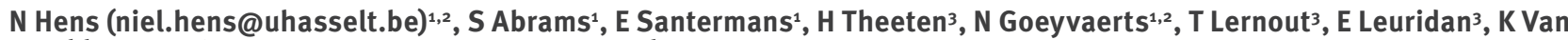

Kerckhove ${ }^{1}$, H Goossens ${ }^{4}$, P Van Damme ${ }^{3}$, P Beutels ${ }^{2,5}$

1. Interuniversity Institute for Biostatistics and statistical Bioinformatics, Hasselt University, Hasselt, Belgium

2. Centre for Health Economic Research and Modelling Infectious Diseases, Vaccine and Infectious Disease Institute (WHO Collaborating (entre), University of Antwerp, Antwerp, Belgium

3. Centre for the Evaluation of Vaccination, Vaccine and Infectious Disease Institute (WHO Collaborating Centre), University of Antwerp, Antwerp, Belgium

4. Laboratory of Medical Microbiology, Vaccine and Infectious Disease Institute (WHO Collaborating Centre), University of Antwerp, Antwerp, Belgium

5. School of Public Health and Community Medicine, The University of New South Wales, Sydney, Australia

Citation style for this article:

Hens N, Abrams S, Santermans E, Theeten H, Goeyvaerts N, Lernout T, Leuridan E, Van Kerckhove K, Goossens H, Van Damme P, Beutels P. Assessing the risk of measles resurgence in a highly vaccinated population: Belgium anno 2013. Euro Surveill. 2015;20(1):pii=20998. Available online: http://www.eurosurveillance. org/ViewArticle.aspx?Articleld=20998

Article submitted on 01 February 2014 / published on 08 January 2015

Despite long-standing two-dose measles-mumpsrubella (MMR) vaccination, measles outbreaks still occur in highly vaccinated European populations. For instance, large measles outbreaks occurred in France (2008-13), the United Kingdom (2012-13) and the Netherlands (2012). Based on a multicohort model approach, using spatial serological survey data, MMR vaccination coverage data and data on social contacts, we found effective reproduction numbers significantly higher than 1 for measles in Belgium. This indicates that at one of the expected re-introductions, a measles outbreak is likely to spread, especially when it occurs during school term. The predicted average effective reproduction number increased over a 30-year time span from 1.3 to 2.2 and from 1.9 to 3.2 for basic reproduction numbers of 12 and 18, respectively. The expected relative measles incidence was highest in infants under one year of age, in adolescents and young adults. In conclusion, gradually increasing proportions of susceptible adolescents and young adults provide through their highly active social life an avenue for measles to resurge in large outbreaks upon re-introduction in Belgium, especially during school terms. Infants form an important vulnerable group during future measles outbreaks.

\section{Introduction}

A large-scale measles outbreak in France started in 2008 , with more than 20,000 reported measles cases by 2013 (see e.g. [1]). In 2012 and 2013, large-scale measles outbreaks have also been reported in the Netherlands [2] and the United Kingdom (UK) [3,4]. To date, no large measles outbreaks have been reported in Belgium since the start of the two-dose vaccination programme in 1995, although some small outbreaks occurred in specific subpopulations. In 2007 and 2008, an outbreak was reported in orthodox Jewish communities [5]. In 2011, a measles outbreak started in a day-care centre and spread to anthroposophic schools, where vaccination coverage was low [6]. It is of interest to determine whether a potential for a resurgence of measles in Belgium still exists. Typically, serological data are used to determine the age-specific susceptibility profile of the population of interest. However, proper quantification of the risk of a possible resurgence based solely on such serological data is only possible if these data are recently collected and if the probability of transmission can be assumed to be independent of age.

Since there are no such recent serological data for Belgium, we apply a newly developed multicohort model [7] that allows using available serological data not necessarily collected at the calendar time of interest. In this approach, the serological data are combined with data on vaccination coverage and data on social contacts. These data are supplemented by estimates of the duration of maternal immunity and of primary and secondary vaccine failure, which were obtained from extensive literature reviews with meta-analysis.

\section{Methods}

We first present the data sources that we relied on, and then briefly introduce the cohort model as proposed in [7].

\section{Data sources}

\section{Serological data}

We used serological data on measles from 2006 in Belgium. Details about data collection and testing can be found in [8]. Briefly, residual samples were collected using a multi-tiered approach to reach a sufficient number of serum samples $(n=3,884)$. To obtain a geographically well-distributed sample, 15 diagnostic laboratories were involved that were spread over 
the country's 10 provinces. They were allocated fixed numbers of samples per age group according to a twostage stratified survey with probability proportional to size with the regions (Flanders, Wallonia, Brussels capital region) in a first stage and the provinces in a second stage. To avoid election of immunosuppressed subjects by using residual samples, specific selection criteria were communicated to the laboratories [8]. For each sample, the birth date, sampling date, sex and postal code of the place of residence were provided by the collecting laboratories. For those samples with missing postal code, the laboratory's postal code was used instead. Samples were analysed with commercial ELISA (Enzygnost, Siemens, Germany). Equivocal results were considered positive.

\section{Vaccination coverage}

Vaccination coverage estimates for Belgium for both recommended measles-mumps-rubella (MMR) doses were taken from [9-18].

Waning of maternally acquired immunity

Newborns are initially protected through maternal antibodies. We used estimates as reported by several authors [19-22].

\section{Primary and secondary vaccine failure}

An extensive literature search in PubMed and Thomson Reuters Web of Knowledge was conducted to obtain estimates for the seroconversion (as a proxy of primary vaccine failure) and exponential waning rates (as a proxy of secondary vaccine failure). Seropositivity for anti-measles IgG was used as a proxy for natural infection or vaccine-induced protection, and seronegativity as a proxy for susceptibility. A proportion of persons who do not have detectable measles antibody may have a level of protection via cellular immunity, but this proportion remains unknown [23] and could thus not be taken into account in our model. Random-effects meta-analyses were carried out to obtain overall estimates. Overviews of the studies included in the metaanalyses are available in the supplementary material (http://ibiostat.be/online-resources)*.

\section{Social contact data}

We used social contact data from Belgium collected in the European study POLYMOD [24] to estimate the agespecific relative incidence of a resurgence of measles. We used social contact data from holiday and schoolterm periods as reported by [25].

Basic reproduction numbers

In the absence of pre-vaccination serological data, our method relies on assuming a specific value for the basic reproduction number $R_{0}$ for measles. We rely on estimates of $R_{0}$ for measles as reported by [26], ranging from 12 to 18 .

\section{Cohort model}

The model we applied was introduced in [7] as a multicohort model that used the most recently available serological information on mumps in a highly vaccinated population such as in Belgium in 2006 to quantify the risk of mumps outbreaks in 2012. While referring to [7] for further methodological details, we can briefly describe the multicohort model according to a three step procedure: (i) modelling the serological data in 2006, (ii) deriving the spatial age-dependent susceptibility profile for 2013 using a cohort model and (iii) using social contact data and the inferred next generation matrix to obtain estimates of the effective reproduction number and the age-dependent relative incidence. In the current analysis, we assume that serological status (seropositivity for anti-measles IgG) is a perfect marker for immunity. Susceptibility therefore refers to seronegativity for anti-measles IgG, seroconversion refers to changing from seronegative to seropositive and waning of immunity refers to IgG antibody decay. We will come back to these assumptions in the discussion.

A model for the serological data in 2006

We used a generalised additive model to estimate measles seroprevalence as a function of age $a$, sex $g$ and spatial location $(x, y)$. The generalised additive model with complementary log-log link function [27] can be formulated as follows:

$$
\operatorname{clog} \log (\pi(a, x, y, g))=f(a, x, y, g),
$$

where $\pi(a, x, y, g)$ represents the proportion of seropositives of age $a$ with spatial coordinates $(x, y)$ and sex $g$, and $f$ is a smooth function. Generalised additive models, extending the well-known generalised linear models, allow for spatial interpolation through the use of scatterplot smoothers resulting in the estimation of a smooth susceptibility profile at the municipality-level. Submodels of model (1), including some or all of the available covariate information (i.e. age, sex and spatial location) were considered and results of fitting these models are presented together with a comparison based on the Akaike information criterion (AIC). The model with the smallest AIC value was retained for the estimation of the age-specific seronegativity to measles in Belgium. The smooth function $f$ was decomposed in smooth components $s_{i}(\cdot), i=1,2$ which were fitted using one-dimensional cubic splines and two-dimensional thin-plate regression splines, respectively, and/or components te $(\cdot, \cdot)$ referring to tensor product thin-plate regression splines allowing for differential smoothing along the two dimensions. As a result, using this approach, geographical estimates of the susceptibility profile in $\mathbf{2 0 0 6}$ are obtained by averaging data points with their neighbours. 
Geographically and age-dependent susceptibility profiles in 2013

Briefly, denote $s_{b}(a)$ the proportion of susceptible individuals of age $a$ born in year $b$. Note that the calendar time can be calculated as $t=b+a$. The multicohort model was based on the following set of equations:

$$
\begin{aligned}
& 1-s_{b}(a)=e^{-\eta \times a} \times\left(1-s_{b}(0)\right) \text { if } 0 \leq a<1, \\
& 1-s_{b}(a)=e^{-\gamma_{1} \times(a-1)} \times \rho v_{1} \text { if } 1 \leq a<12, \\
& 1-s_{b}(a)=e^{-\gamma_{2} \times(a-12)} \times \rho v_{2} \text { if } 12 \leq a .
\end{aligned}
$$

Here $\rho$ represents the seroconversion rate, $y_{1}$ and $y_{2}$ the decay rates of vaccine-induced immunity related to dose 1 and $2, s_{b}(0)$ is the proportion of susceptible newborns (informed by the fraction of susceptible women of childbearing age) and $n$ the rate at which maternal antibodies decay (3.87 year $^{-1}$, see [7]). Given that our interest was in calculating the age-dependent proportion of susceptible individuals at calendar time 2013, we needed to adapt and apply the aforementioned cohort model in the following way: Firstly, for individuals 20 years and older (who were 13 years or older in 2006) we needed to take vaccine and naturally induced immunity into account. We did this by combining the estimated proportion of susceptible persons in the 2006 serological data with estimates of the vaccination coverage (MMR second dose) over time. The age-dependent proportion of susceptible persons was estimated by using a generalised additive model with a radial spline for age. To propagate this estimate to future years we took into account waning vaccineinduced immunity (multiplication with $e^{-y 2 \times(a-a o)}$, with $a_{o}$ the age in the year 2006) and relied on lifelong immunity following natural infection. Secondly, for individuals younger than 20 years, we could not use the 2006 serological data to estimate the proportion susceptible because a second MMR dose had been offered to these children afterwards and we needed to rely entirely on the more recent vaccination coverage data. We could then use equation set (2) to determine the agedependent proportion of susceptibles. Note that we also adapted equation set (2) to account for groups of individuals that received the first dose only.

\section{The reproduction number and age-dependent relative} incidence

The basic/effective reproduction number is the expected number of secondary cases produced by a typical infected individual during their entire infectious period when introduced into a completely/partially susceptible population. The basic/effective reproduction number determines the spread of the virus in the population: if it is lower than 1, the virus will stop spreading; if it is higher than 1, the virus will spread. Based on the age-dependent susceptibility profile, social contact data and a literature-based estimated range for $R_{0}$, we calculated the effective reproduction number $R$, and the age-specific relative incidence of re-emerging measles outbreaks in Belgium. We used social contact data from holiday and school-term periods to infer the effective reproduction number in each
TABLE 1

Generalised additive models fitted to seroprevalence data on measles infection with corresponding AIC values, Belgium, 2006

\begin{tabular}{|l|c|c|}
\hline Model & Linear predictor & AIC \\
\hline$(1)$ & $t e(x, y, a, b y=g)+t e(x, y, b y=1-g)$ & $1,098.42$ \\
\hline$(2)$ & $t e(x, y, a)$ & $1,091.86$ \\
\hline$(3)$ & $s_{1}(a)+t e(x, y)$ & $1,085.68$ \\
\hline$(4)$ & $S_{1}(a)+S_{2}(x, y)$ & $1,085.66$ \\
\hline$(5)$ & $S_{1}(a)$ & $1,126.24$ \\
\hline
\end{tabular}

AIC: Akaike information criterion.

$a$ is age, $x, y$ are spatial coordinates and $g$ is sex (o/1 - female/ male).

of those periods. Note that we did not use spatially adjusted contact patterns given that sufficient information to obtain regional contact patterns was not available. As a result, contact patterns were assumed spatially invariant.

Uncertainty

Uncertainty was taken into account by applying a parametric bootstrap which enabled us to calculate $95 \%$ confidence intervals $(\mathrm{Cl})$ for the effective reproduction number using contact patterns from both holiday and school-term period and assuming $\mathrm{R}_{0}$ equal to either 12 or 18 (using school-term period contact patterns). Furthermore, uncertainty related to vaccination coverage was taken into account by resampling vaccination coverage information from the available $95 \% \mathrm{Cls}$ through the specification of underlying normal distributions with corresponding percentiles, from which random samples were drawn. An interpolating spline model was used to obtain a smooth susceptibility profile as well as $95 \%$ Cls.

\section{Results}

Table 1 summarises the result of modelling the serological data for 2006. The generalised linear model consisting of an additive decomposition of age and spatial coordinates was the preferred model based on AIC (1,085.66). The second best model has a slightly higher AIC $(1,085.68)$ and used a tensor-product spline (te) which yielded differential smoothing for the spatial coordinates $x$ and $y$ as compared with the best model $(s(\cdot, \cdot))$. Other models resulted in substantially higher (i.e. comparatively worse) AIC values.

Our random-effects meta-analyses resulted in an estimated $\rho$ or seroconversion rate of $0.977(95 \% \mathrm{Cl}$ : 0.959-0.990) and estimated $\gamma_{1}$ and $\gamma_{2}$, the exponential waning rates of $0.007(95 \% \mathrm{Cl}: 0.003-0.018)$ after the first $(d=1)$ and of $0.008(95 \% \mathrm{Cl}: 0.004-0.020)$ after the second $(d=2)$ dose of the trivalent MMR vaccine. Based on these results, a common $\gamma$ or waning rate for 


\section{FIGURE 1}

Age-specific susceptibility to measles infection, Belgium, 2013
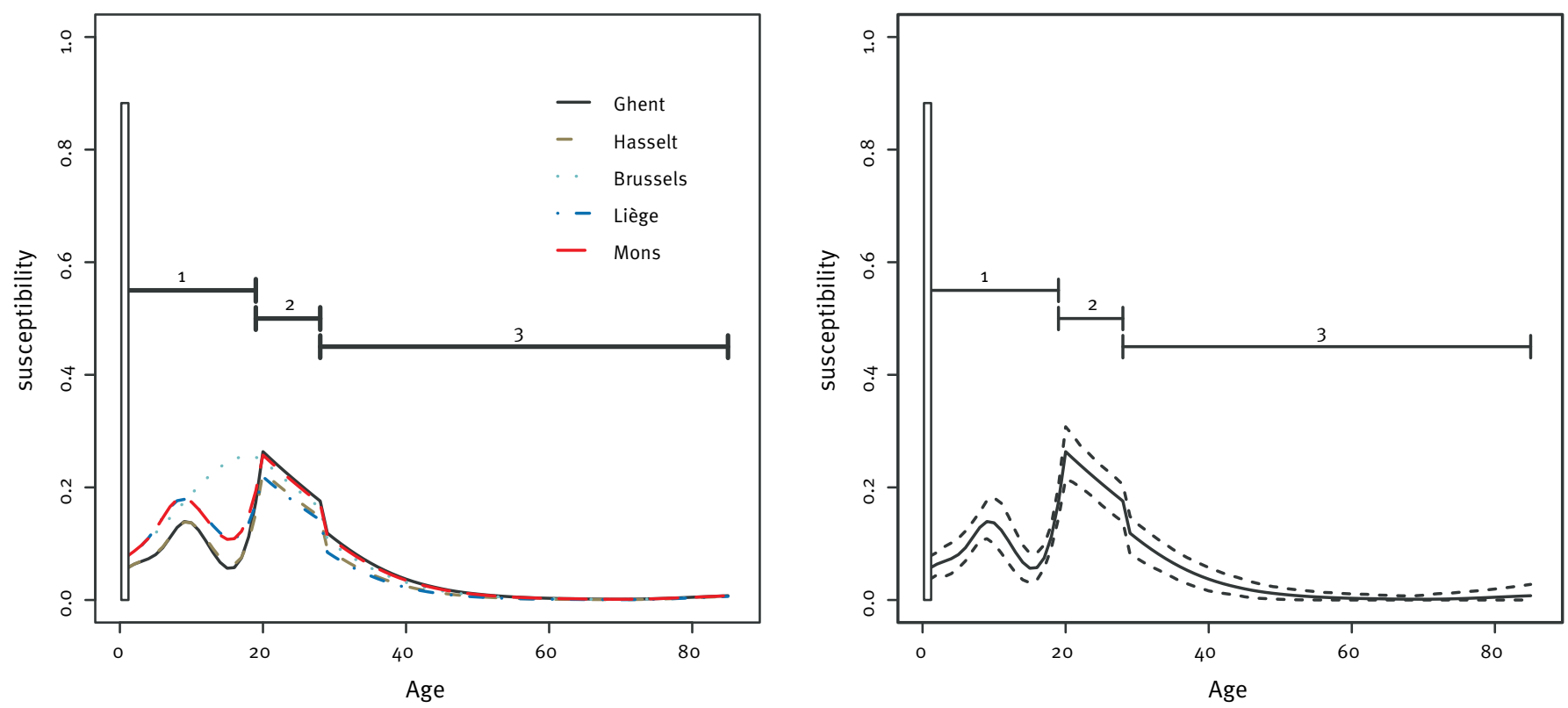

Left panel: estimated susceptibility in five Belgian cities.

Right panel: Estimated susceptibility (solid line) and $95 \%$ confidence limits (dashed lines) for Ghent.

The susceptibility curve is based on (i) coverage information assuming waning of vaccine-induced immunity; (ii) coverage information, serology and assuming waning of vaccine-induced immunity; and (iii) serology assuming lifelong natural immunity. The bar on the left hand side represents the proportion of susceptible infants younger than one year.

TABLE 2

Estimated vaccination coverages for MMR in per region and 95\% confidence intervals when available, Belgium survey years 1995-2012

\begin{tabular}{|c|c|c|c|c|}
\hline \multirow{2}{*}{$\begin{array}{l}\text { Year and } \\
\text { Region }\end{array}$} & \multicolumn{2}{|c|}{ MMR first dose } & \multicolumn{2}{|c|}{ MMR second dose } \\
\hline & Estimated coverage in $\%$ & $95 \% \mathrm{Cl}$ & Estimated coverage in $\%$ & $95 \% \mathrm{Cl}$ \\
\hline \multicolumn{5}{|l|}{1995} \\
\hline \multicolumn{5}{|l|}{1999} \\
\hline Flanders & 83.4 & $80.3-86.5$ & NA & NA \\
\hline Wallonia & 82.4 & NA & NA & NA \\
\hline \multicolumn{5}{|l|}{2000} \\
\hline Brussels & $74 \cdot 5$ & $70.1-78.9$ & NA & NA \\
\hline \multicolumn{5}{|l|}{2003} \\
\hline Wallonia & 82.5 & NA & NA & NA \\
\hline \multicolumn{5}{|l|}{2005} \\
\hline Flanders & 94.0 & $92.6-95.3$ & 83.6 & $81.4-85.8$ \\
\hline \multicolumn{5}{|l|}{2006} \\
\hline Brussels & 91.1 & $88.7-93.6$ & 70.5 & NA \\
\hline Wallonia & 89.0 & $86.3-91.8$ & 70.5 & NA \\
\hline \multicolumn{5}{|l|}{2008} \\
\hline Flanders & 96.6 & $95.2-97.6$ & 90.6 & $89.0-92.2$ \\
\hline \multicolumn{5}{|l|}{2009} \\
\hline Wallonia & 92.4 & $90.2-94.6$ & $75 \cdot 5$ & NA \\
\hline \multicolumn{5}{|l|}{2012} \\
\hline Brussels & 94.1 & $92.1-96.1$ & $\mathrm{NA}$ & $\mathrm{NA}$ \\
\hline Flanders & 96.6 & $95.1-97.6$ & 92.5 & $90.9-94.1$ \\
\hline Wallonia & 94.4 & $92.4-96.4$ & NA & NA \\
\hline
\end{tabular}

$\mathrm{Cl}$ : confidence interval; MMR: measles-mumps-rubella vaccine; NA: not applicable. 
Time-specific estimated effective reproduction numbers for measles: spatial average and averaged 95\% CI, Belgium, 2013
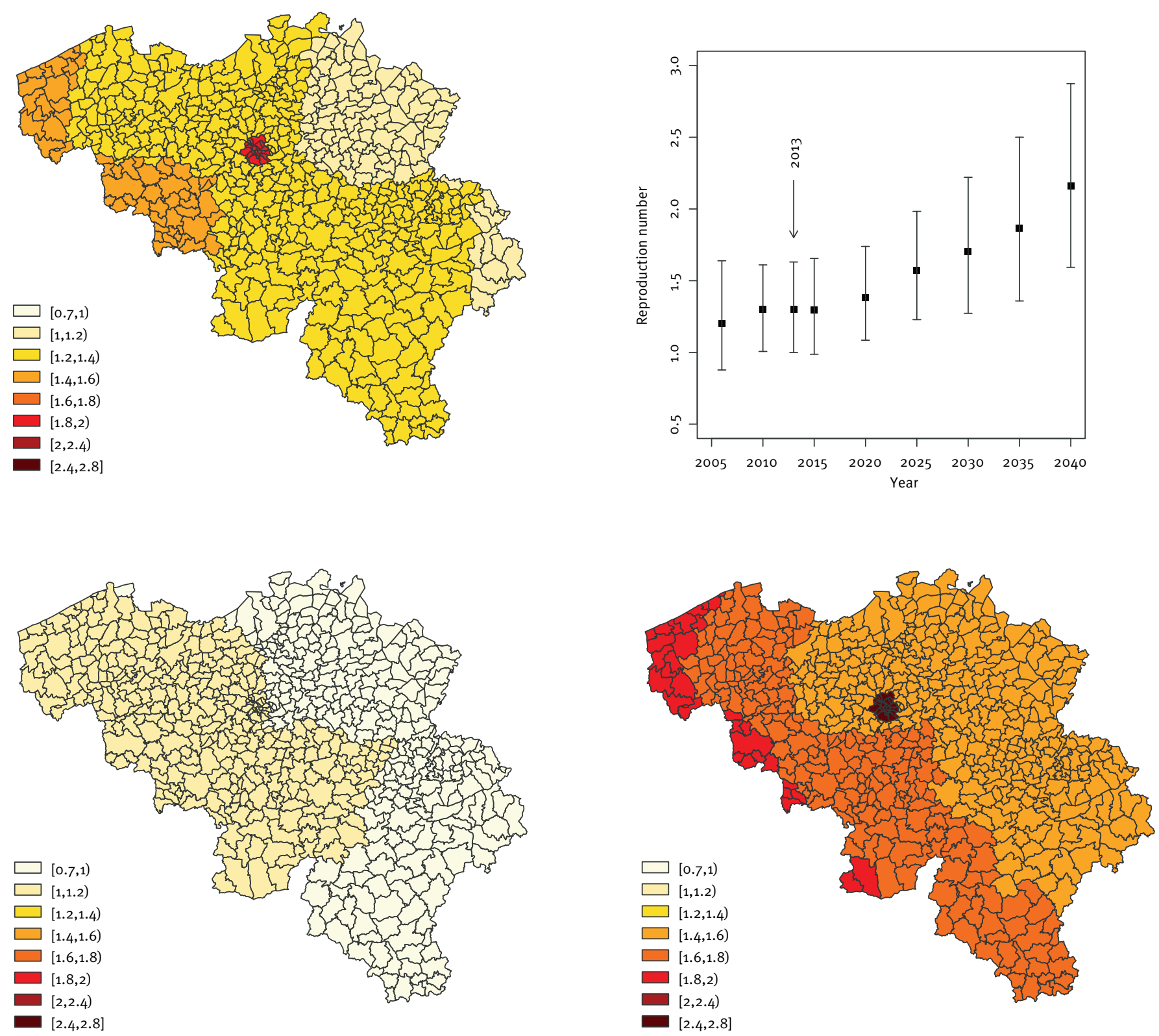

Upper left panel: Estimated effective reproduction numbers for a school-term period and Ro $=12$ for Belgium in 2013. Upper right panel: Timespecific estimated effective reproduction numbers for Belgium: spatial average and averaged $95 \%$ Cl. Bottom panels: $95 \%$ confidence limits for effective reproduction numbers for a school-term period and Ro $=12$ for Belgium in 2013.

doses 1 and 2, equal to 0.008 (95\% Cl: 0.005-0.014), was assumed.

Figure 1 (left panel) shows the age-specific susceptibility profile in 2013 for five Belgian cities. The susceptibility curve was based on different data sources and assumptions: (i) coverage information summarised in Table 2, and waning of vaccine-induced immunity; (ii) coverage information, serology and waning of vaccineinduced immunity; and (iii) serology with lifelong natural immunity. Susceptibility among adolescents was high but decreased after vaccination at 12 months and 12 years of age. The susceptibility curve for Brussels was somewhat distinct from those for Ghent, Hasselt, Liège and Mons as a result of limited historical information on vaccination coverages in Brussels (Table 2). Figure 1 (right panel) shows the age-specific susceptibility profile for Ghent together with $95 \% \mathrm{Cl}$.

The spatial pattern of effective reproduction numbers for Belgium during a school-term period in 2013 and assuming $R_{0}=12$ is shown in the upper left panel of 
Boxplots of the spatial distribution of effective reproduction numbers for measles infection assuming $\mathrm{R}_{0}=12$ (left column) and $\mathrm{R}_{0}=18$ (right column), Belgium, 2013

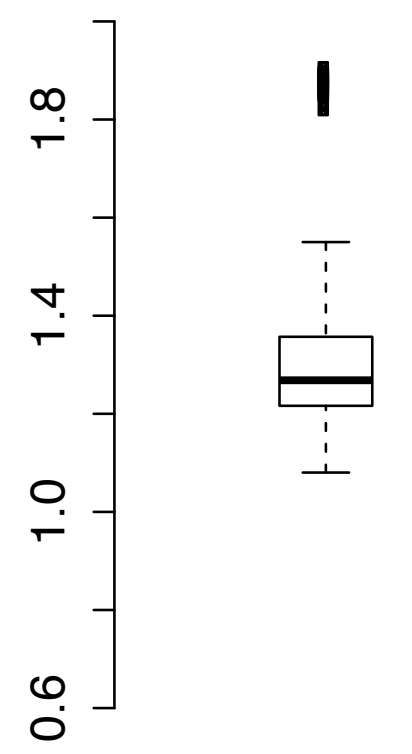

School term

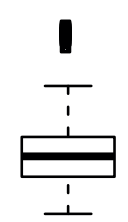

Holiday

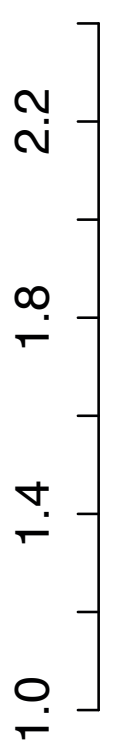

School term

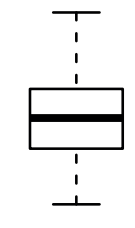

Holiday

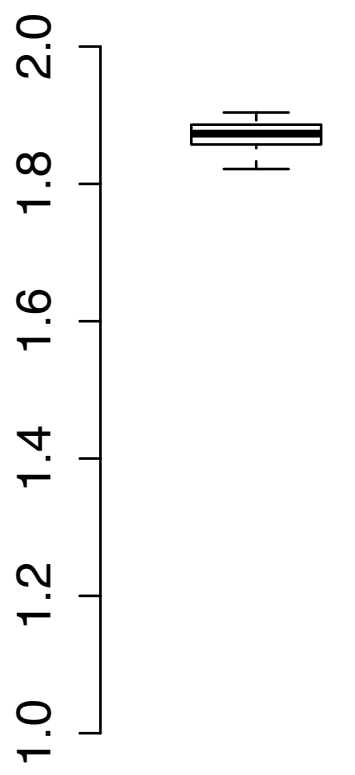

Brussels

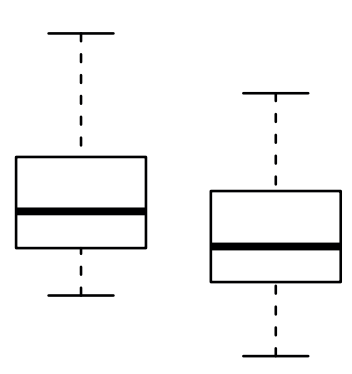

Wallonia

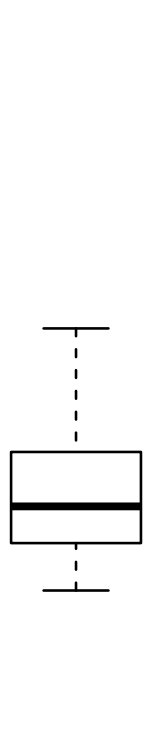

Flanders

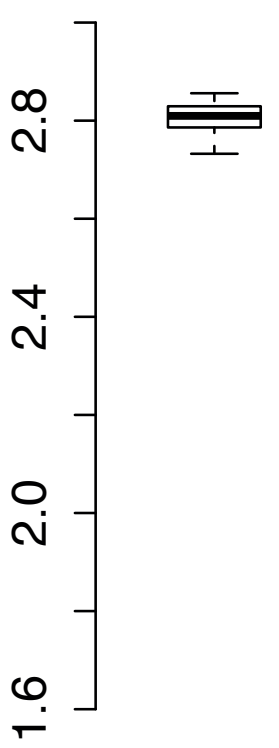

Brussels
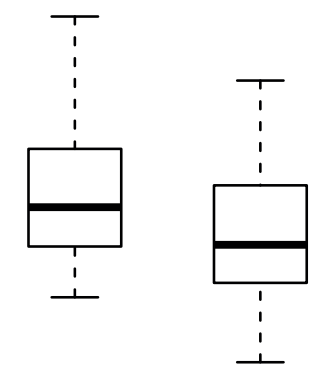

Flanders

Differences between school-term and holiday periods (upper row) and differences between the three Belgian regions (bottom row) in 2013.

Figure 2. The corresponding $95 \% \mathrm{Cl}$ are shown in the two bottom panels of Figure 2. In the upper right panel of Figure 2, the corresponding averaged $95 \% \mathrm{Cl}$ and average effective reproduction number are shown over time for $R_{0}=12$. The temporal change in the effective reproduction number already exceeded the epidemiological threshold in 2006, if non-significantly, whereas for 2010, the reproduction number had an estimated value which was significantly above 1 . After 2010, the predicted average effective reproduction number increased over a 30-year time span from ca 1.3 to 2.2. Results for $R_{0}=18$ yielded a similar spatial pattern with effective reproduction numbers ranging from ca 1.6 to 2.4 with an average of ca 1.9 (Figure 3, upper right panel, first box plot). The predicted average effective reproduction number increased over 30 years from 1.9 to 3.2 (not shown).

Figure 3 shows boxplots of location-specific effective reproduction numbers for a school-term period and a holiday period in the upper left panel $\left(R_{0}=12\right)$ and upper right panel $\left(R_{0}=18\right)$. These results clearly demonstrated a substantial reduction in outbreak risk during holiday periods and a decrease of the majority of 
effective reproduction numbers below 1 when $R_{0}=12$. The bottom left panel and the bottom right panel of Figure 3 show substantial differences between the Brussels capital region and the two other regions in Belgium that are mainly due to a lower vaccination coverage (and more limited historical information) in the Brussels capital region and to a lesser extent also due to differences in observed seropositivity between the different regions.

Figure 4 shows the expected age-specific relative incidence of a measles epidemic upon re-introduction of measles in the Belgian population for five different cities: Brussels, Ghent, Hasselt, Liège and Mons in a school-term period. A large proportion of new cases is expected to occur in infants younger than one year because of rapid waning of maternal antibodies [21,22]. The peak incidence for people older than one year is expected in the age category 20 to 24 years, except for Brussels where the peak incidence is expected in the age category 15 to 19 years. Note that because of a higher expected absolute incidence in the age categories 15 to 19 and 20 to 24 years in Brussels compared with the other four cities, the relative incidence for infants younger than one year was estimated lower in Brussels compared with the other four cities. This observation and the peak incidence at 15 to 19 and 20 to 24 years resulted from an increased susceptibility in 2013 of the age categories 15 to 19 and 20 to 24 years in Brussels and the other cities, respectively and a higher frequency of social contact in the 15 to 19 yearolds $[24,28]$. Taking both susceptibility and contact intensity into account is important to explain the relatively high expected incidence in the 15 to 19 year age category for all five cities, whereas the susceptibility in 2013 in this age category was estimated lower than in the age category 20 to 30 years, due to vaccination in the intermittent years (Figure 1).

\section{Discussion}

Using a simple multicohort model [7], we were able to estimate current spatially-explicit age-dependent measles outbreak risk using seroprevalence data, vaccine coverage data and social contact data from Belgium. Our main findings can be summarised as follows: (i) outbreak risk (effective reproduction number $>1$ ) exists all over the country, at least in school-term periods, so imported infections have the potential to spread; (ii) higher risk exists in school-term periods than in holiday periods, implying an increase in risk every time school starts compared with the corresponding preceding holiday period; (iii) at highest risk are infants under one year of age, adolescents and young adults; (iv) spatial heterogeneity in outbreak risk is observed but should not be overinterpreted given that considerable uncertainty exists; ( $v$ ) propagating predictions based on the most-recently observed vaccination coverages shows that the effective reproduction number is expected to increase over the next few decades.
FIGURE 4

Predicted age-specific relative incidence for newly emerging measles outbreaks in five Belgian cities: Brussels, Ghent, Hasselt, Liège and Mons, 2013

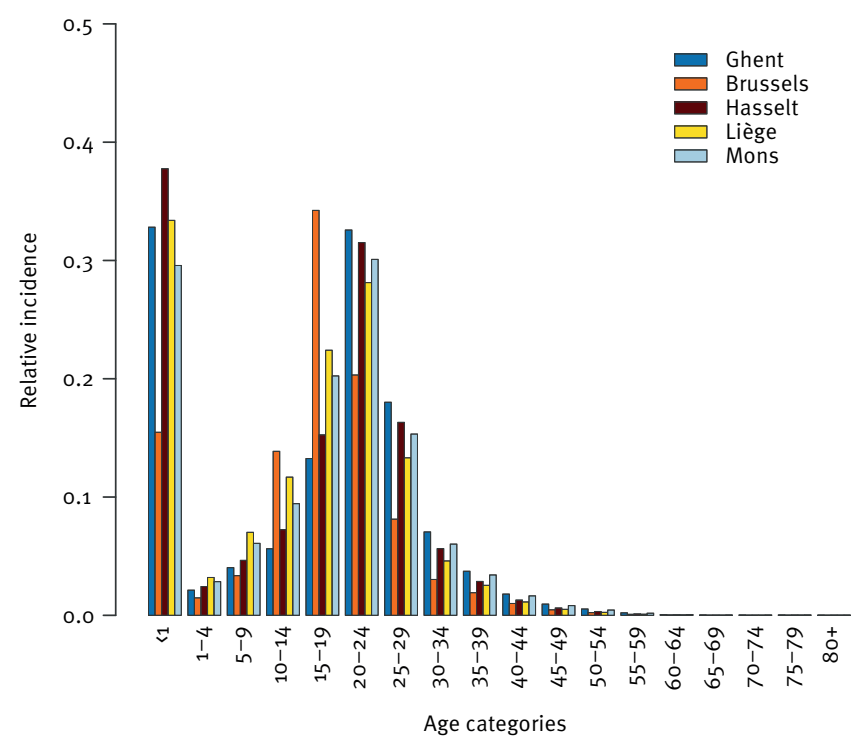

Despite recent large measles outbreaks in the Netherlands, France and the UK, all neigh- bouring countries of Belgium, no new measles outbreaks have been reported since the small local epidemic in 2011 in Belgium. Only 39 isolated cases were reported in 2013, corresponding to an incidence of 3.5 per million inhabitants (personal communication: Martine Sabbe, Scientific Institute of Public Health, Brussels, January 2014). However, even within the Netherlands, the outbreak did not spread to the wider community. Instead it affected mostly the Dutch Bible Belt, which consists of a large cluster of families with low vaccination coverage (from $<80 \%$ to $90-95 \%$ [29]), inspired by their religious beliefs. The epidemic had not (yet) spread to the surrounding highly vaccinated regions, including the Belgian-Dutch border. In addition, with effective reproduction numbers slightly above 1 , potential outbreaks go extinct very rapidly. For example, assuming homogeneous mixing and an effective reproduction number equal to 1.3 would result in ca $62 \%$ of outbreaks going extinct with a final size smaller than $n=10$ [30].

School outbreaks of measles have been reported very often, and seasonal variation with a clear impact of school holidays has been demonstrated in the period before mass vaccination against measles was launched [31,32]. In those days, mainly children were at risk for measles outbreaks. The projected outbreak risk in Belgium increases as a result of simultaneous processes involving two large subgroups of the population. On the one hand, vaccine-induced immunity among vaccinated people wanes as they age over time. On the other hand, the generally older subgroup of incomplete or unvaccinated people, many of whom acquired long-lasting immunity following natural 
infection, grows older, and thus on average has fewer social contacts and a higher probability of all-cause death over time. That means that the proportion of the population that is naturally protected from measles is gradually dying out. These processes imply that the overall susceptibility and outbreak risk increase over time. This risk is highest in the part of the population that typically constitutes the engine of airborne transmission through their social contacts at childcare, school, and within and between households. In order to achieve the European measles elimination goal, public health authorities in a number of countries are undertaking campaigns to raise MMR second dose coverage in adolescents which is currently still below the 95\% target needed for elimination (see Table 2). Young adults are, however, harder to reach in such campaigns, and sporadic preventive health services in Belgium are often limited to working or student populations. Suboptimally vaccinated cohorts of young adults could thus delay the impact of elimination efforts. A catchup campaign focusing all young adults is necessary to achieve elimination in the short term.

Our analysis showed that using a relatively simple model [7] and data that are commonly available for most European countries, the outbreak risk for measles can be estimated and age groups in which the risk is highest can be identified. Our method differs from the one used for the analysis of Australian serosurvey data in 2012 [33] by accounting for secondary vaccine failure and using social contact data while acknowledging spatial heterogeneity in susceptibility and vaccination coverage. Our study has several limitations: Firstly, we relied on antibody seropositivity as a proxy for immunological protection against measles infection based on an ELISA test [23]. The ELISA used to detect measles antibodies (Enzygnost, Siemens, Germany) was, as most diagnostic tests, more specific than sensitive $(100 \%$ and $99.6 \%$, respectively, as reported by the manufacturer), which may have overestimated susceptibility at the population level. Moreover, ELISA results do not perfectly correlate with the more sensitive plaque reduction neutralisation values $(>120)$ which were used in few other existing studies to relate circulating antibodies to clinical protection from measles, e.g. [34]. Antibodies are the main but not the only mechanism of immunological protection. For instance, long-lasting immunity after natural measles infection in patients with deficient humoral immunity (primary agammaglobulinaemia) has been demonstrated, indicating that the cellullar immune system alone is capable of preventing measles. Therefore, again, susceptibility might be overestimated if based on antibody titres only. On the other hand, in the current study, equivocal results were classified as positive and therefore could result in underestimating susceptibility at the population level. However, the age and spatial patterns are likely to have remained unaffected by this. Secondly, the spatial resolution of various data we used was limited. Nevertheless, the analysis of the serological data clearly indicated spatial heterogeneous serological profiles. Coverage data (and their spatial resolution) were not available to the same extent in each region, and the spatial distribution of our social contact data was not detailed enough due to sample size restrictions, limiting spatial estimates of susceptibility. Thirdly, susceptibility was only allowed to change over time as a result of waning of vaccine-induced immunity, and therefore, very rarely, decreases in susceptibility caused by sporadic infections over the years are ignored. Finally, our method relied on several inputs such as a realistic estimate of the basic reproduction number $R_{0}$ in the study population and estimates for the waning rates after vaccination for which uncertainty was accounted for in the parametric bootstrap approach.

Although the demographic structure of a population is of importance in the estimation of the effective reproduction number and varies over time, we did not use a dynamic model in which population and infectious disease dynamics were modelled simultaneously. Our method was based on a cohort model, which could be adjusted for different population structures; this is deemed necessary especially if the serological survey sampling occurs long before the time point of interest. Therefore, prediction of the effective reproduction number over time relied on observed population sizes and predicted population sizes for future years according to a demographic model for Belgium (data not shown). The proposed methodology can be extended to include diagnostic test uncertainty, which was not pursued here because of a specificity and sensitivity close to $100 \%$. It can also be used as an informative pilot for the design of studies to document serological profiles, social contacts and vaccination coverage in populations with the aim to improve outbreak risk assessments.

Our model has partly been validated by (i) the mumps outbreak in 2012 in Flanders for which, in hindsight, our estimates are in line with the observed incidence [35], (ii) a small measles outbreak in a day care centre in the province of Antwerp for which the incidence was in line with the predicted relative incidence [36]. Indeed, young infants remain at increased risk for measles since maternal antibodies are waning rapidly during the first months of life. Almost all cases in that outbreak occurred in infants too young to be vaccinated with the first MMR dose. These cases can be prevented by vaccination strategies targeting the other susceptible age groups in society. The currently available measles vaccines are not effective enough when routinely used in infants younger than one year.

Current vaccination campaigns focus on improving vaccination coverage especially for the second dose of MMR at its recommended age (10 to 13 years). In Flanders, schools and vaccinating physicians have been encouraged in 2013 and 2014 to promote MMR2 and reduce missed vaccination opportunities, and 
further actions to better reach underserved populations are foreseen.

Given the high relevance of our results for public health, the competent authorities were informed about our work and a press release based on the findings of this study has been released at the start of the 2013/14 campaign mentioned above. In that press release we advised people aged 20 to 30 years to check their vaccination status and take action if it was incomplete. Official recommendations for catch-up vaccination with MMR have been updated and include adults up to birth year 1971 , but to date, no national or regional campaigns have been undertaken to increase coverage in this age group.

\section{${ }^{\star}$ Note}

Supplementary information made available by the authors on an independent website is not edited by Eurosurveillance, and Eurosurveillance is not responsible for the content. The material can be accessed at: http://ibiostat.be/ online-resources/.

\section{Acknowledgements}

The authors would like to acknowledge the colleagues of the Flemish Agency for Care and Health, the Scientific Institute of Public Health and the measles elimination committee for fruitful discussions related to this research. NH acknowledges support of the University of Antwerp Scientific Chair in Evidence-based Vaccinology sponsored in 2009-2014 by a gift from Pfizer. SA acknowledges support by the Research Fund of Hasselt University (Grant BOF11NI31). ES and HG acknowledge support from a Methusalem research grant from the Flemish government. NG is beneficiary of a postdoctoral grant from the AXA Research Fund. Support from the IAP Research Network P7/06 of the Belgian State (Belgian Science Policy) is gratefully acknowledged. HT and EL are postdoctoral researchers for the Fund of Scientific Research - Flanders (FWO). The computational resources and services used in this work were provided by the Hercules Foundation and the Flemish Government - department EWI.

\section{Conflict of interest}

None declared.

\section{Authors' contributions}

$\mathrm{NH}, \mathrm{SA}$ and ES performed the analyses. HT, EL, TL and KVK provided the data for the analyses and contributed together with NG, HG, PVD and PB to the analyses and their interpretation.

$\mathrm{NH}, \mathrm{SA}$ and $\mathrm{PB}$ wrote the initial draft of the manuscript after which all authors contributed to drafting the final manuscript. All authors read and approved the final manuscript.

\section{References}

1. Antona D, Lévy-Bruhl D, Baudon C, Freymuth F, Lamy M, Maine $C$, et al. Measles elimination efforts and 2008-2011 outbreak,
France. Emerg Infect Dis. 2013;19(3):357-64. http://dx.doi. org/10.3201/eid1903.121360 PMID:23618523

2. Knol M, Urbanus A, Swart E, Mollema L, Ruijs W, van Binnendijk R, et al. Large ongoing measles outbreak in a religious community in the Netherlands since May 2013. Euro Surveill. 2013;18(36):20580. http://dx.doi.org/10.2807/15607917.ES2013.18.36.20580 PMID:24079377

3. Vivancos R, Keenan A, Farmer S, Atkinson J, Coffey E, Dardamissis E, et al. An ongoing large outbreak of measles in Merseyside, England, January to June 2012. Euro Surveill. 2012;17(29): $\mathrm{pii}=20226$. PMID:22835470

4. Baugh V, Figueroa J, Bosanquet J, Kemsley P, Addiman S, Turbitt D. Ongoing measles outbreak in Orthodox Jewish community, London, UK. Emerg Infect Dis. 2013;19(10):1707-9. http://dx.doi.org/10.3201/eid1910.130258 PMID:24050804

5. Lernout T, Kissling E, Hutse V, De Schrijver K, Top G. An outbreak of measles in orthodox Jewish communities in Antwerp, Belgium, 2007-2008: different reasons for accumulation of susceptibles. Euro Surveill. 2009;14(2): pii=19087. PMID:19161716

6. Braeye T, Sabbe M, Hutse V, Flipse W, Godderis L, Top G. Obstacles in measles elimination: an in-depth description of a measles outbreak in Ghent, Belgium, spring 2011. Arch Public Health. 2013;71(1):17. http://dx.doi.org/10.1186/0778-7367-7117 PMID:23834074

7. Abrams S, Beutels P, Hens N. Assessing mumps outbreak risk in highly vaccinated populations using spatial seroprevalence data. Am J Epidemiol. 2014;179(8):1006-17. http://dx.doi. org/10.1093/aje/kwu014 PMID:24573540

8. Theeten $\mathrm{H}$, Hutse $\mathrm{V}$, Hens $\mathrm{N}$, Yavuz $\mathrm{Y}$, Hoppenbrouwers $\mathrm{K}$, Beutels $\mathrm{P}$, et al. Are we hitting immunity targets? The 2006 age-specific seroprevalence of measles, mumps, rubella, diphtheria and tetanus in Belgium. Epidemiol Infect. 2011;139(4):494-504. http://dx.doi.org/10.1017/ So950268810001536 PMID:20587123

9. Theeten H, Hens N, Vandermeulen C, Depoorter AM, Roelants $M$, Aerts $M$, et al. Infant vaccination coverage in 2005 and predictive factors for complete or valid vaccination in Flanders, Belgium: an EPI-survey. Vaccine. 2007;25(26):4940-8. http:// dx.doi.org/10.1016/j.vaccine.2007.03.032 PMID:17524528

10. Theeten $H$, Vandermeulen C, Roelants $M$, Hoppenbrouwers K, Depoorter AM, Van Damme P. Coverage of recommended vaccines in children at 7-8 years of age in Flanders, Belgium. Acta Paediatr. 2009;98(8):1307-12. http://dx.doi.org/10.1111/ j.1651-2227.2009.01331.x PMID:19432835

11. Vellinga A, Depoorter AM, Van Damme P. Vaccination coverage estimates by EPI cluster sampling survey of children (18-24 months) in Flanders, Belgium. Acta Paediatr. 2002;91(5):599603. http://dx.doi.org/10.1111/j.1651-2227.2002.tb03283.x PMID:12113332

12. Robert E., Swennen B. Enquête de couverture vaccinale des enfants de 18 à 24 mois en Région de Bruxelles-Capitale, 2006. [Vaccination coverage survey in infants at 18-24 months of age in the Brussels Capital Region, 2006]. Brussels: Université Libre de Bruxelles, Ecole de santé publique. Décembre 2006. French. Available from: http://www. observatbru.be/documents/graphics/rapports-externes/ enquete-de-couverture-vaccinale-des-enfants-de-18-a-24mois-en-region-de-bruxelles-capitale.pdf

13. Robert E, Swennen B. Enquête de couverture vaccinale des enfants de 18 à 24 mois en communauté française (Bruxelles excepté), 2009. [Vaccination coverage survey in infants at 18-24 months of age in the French Community except Brussels, 2009]. Brussels: Provac, Université Libre de Bruxelles; Nov 2009. French. Available from: http://ddata.over-blog.com/ xxxyyy/3/27/09/71/Enquete-Provac-2009.pdf

14. Vandermeulen C, Roelants $M$, Theeten H, Depoorter AM, Van Damme P, Hoppenbrouwers K. Vaccination coverage in 14-year-old adolescents: documentation, timeliness, and sociodemographic determinants. Pediatrics. 2008;121(3):e428 34. http://dx.doi.org/10.1542/peds.2007-1415 PMID:18310163

15. Hoppenbrouwers K, Vandermeulen C, Roelants M, Boonen M, Van Damme $\mathrm{P}$, Theeten $\mathrm{H}$, et al. Studie van de vaccinatiegraad bij jonge kinderen en adolescenten in Vlaanderen in 2008. [Vaccination coverage in infants and adolescents in Flanders in 2008]. Brussels: Flemish Governement (Agency of Care and Health), 2009. Dutch.

16. Van Damme P, Theeten H, Braeckman T, Lernout T, Hens $N$, Hoppenbrouwers K, et al. Studie van de vaccinatiegraad bij jonge kinderen en adolescenten in Vlaanderen in 2012. [Vaccination coverage in infants and adolescents in Flanders in 2012]. Brussels: Vlaamse overheid, Vlaams Agentschap Zorg en Gezondheid, Team Infectieziekten; 2013. Dutch.

17. Robert E, Swennen B. Enquête de couverture vaccinale des enfants de 18 à 24 mois en Région de Bruxelles-Capitale, 2012. [Infant vaccination coverage at 18-24 months of age in the Brussels Capital Region, 2012]. Brussels: Université Libre de 
Bruxelles, Ecole de santé publique; Dec 2012. French. Available from: http://www.observatbru.be/documents/graphics/ rapports-externes/enquete-vaccination-2012-fr.pdf

18. Robert E, Swennen B. Enquête de couverture vaccinale des enfants de 18 à 24 mois en Fédération Wallonie-Bruxelles (excepté Bruxelles). [Vaccination coverage survey in infants at 18-24 months of age in the French Community (except Brussels)]. Brussels: Provac, Université Libre de Bruxelles; Dec 2012. French.

19. Sato H, Albrecht P, Reynolds DW, Stagno S, Ennis FA. Transfer of measles, mumps, and rubella antibodies from mother to infant. Its effect on measles, mumps, and rubella immunization. Am J Dis Child. 1979;133(12):1240-3. http://dx.doi.org/10.1001/archpedi.1979.02130120032005 PMID:229727

20. Nicoara C, Zäch K, Trachsel D, Germann D, Matter L. Decay of passively acquired maternal antibodies against measles, mumps, and rubella viruses. Clin Diagn Lab Immunol. 1999;6(6):868-71. PMID:10548578

21. Leuridan E, Hens N, Hutse V, leven M, Aerts M, Van Damme P. Early Waning of Maternal Measles Antibodies in the Era of Global Measles Vaccination. British Medical Journal. 2010;340:C1626.

22. Waaijenborg S, Hahné SJM, Mollema L, Smits GP, Berbers GAM, van der Klis FRM, et al. Waning of maternal antibodies against measles, mumps, rubella, and varicella in communities with contrasting vaccination coverage. J Infect Dis. 2013;208(1):106. http://dx.doi.org/10.1093/infdis/jit143 PMID:23661802

23. Plotkin SA. Complex correlates of protection after vaccination. Clin Infect Dis. 2013;56(10):1458-65. http://dx.doi.org/10.1093/ cid/cito48 PMID:23386629

24. Mossong J, Hens N, Jit M, Beutels P, Auranen K, Mikolajczyk $\mathrm{R}$, et al. Social contacts and mixing patterns relevant to the spread of infectious diseases. PLoS Med. 2008;5(3):e74. http://dx.doi.org/10.1371/journal.pmed.0050074 PMID:18366252

25. Hens N, Ayele GM, Goeyvaerts N, Aerts M, Mossong J, Edmunds JW, et al. Estimating the impact of school closure on social mixing behaviour and the transmission of close contact infections in eight European countries. BMC Infect Dis. 2009;9(1):187. http://dx.doi.org/10.1186/1471-2334-9-187 PMID:19943919

26. Fine PE. Herd immunity: history, theory, practice. Epidemiol Rev. 1993;15(2):265-302. PMID:8174658

27. Wood SN. Generalized additive models: an introduction with R. Boca Raton, Florida: Chapman and Hall/CRC Press; 2006.

28. Hens N, Goeyvaerts N, Aerts M, Shkedy Z, Van Damme P, Beutels $P$. Mining social mixing patterns for infectious disease models based on a two-day population survey in Belgium. BMC Infect Dis. 2009;9(1):5. http://dx.doi.org/10.1186/1471-2334-95 PMID:19154612

29. van Lier EA, Oomen PJ, Giesbers $H$, Conyn-van Spaendonck MAE,Drijfhout IH, Zonnenberg-Hoff IF, et al. Vaccinatiegraad Rijksvaccinatieprogramma Nederland, Verslagjaar 2014 [Coverage of the vaccination programme in the Netherlands, 2014]. Rijksinstituut voor Volksgezondheid en Milieu (RIVM). Dutch. Available from: http://www.rivm.nl/bibliotheek/ rapporten/150202003.pdf

30. Anderson H, Britton T. Stochastic epidemics in dynamic populations: quasi-stationarity and extinction. J Math Biol. 2000;41(6):559-80. http://dx.doi.org/10.1007/s002850000060 PMID:11196585

31. Fine PE, Clarkson JA. Measles in England and Wales--I: An analysis of factors underlying seasonal patterns. Int J Epidemiol. 1982;11(1):5-14. http://dx.doi.org/10.1093/ije/11.1.5 PMID:7085179

32. Word DP, Young JK, Cummings DA, Iamsirithaworn S, Laird CD. Interior-point methods for estimating seasonal parameters in discrete-time infectious disease models. PLoS ONE. 2013;8(10):e74208. http://dx.doi.org/10.1371/journal. pone.0074208 PMID:24167542

33. Gidding HF, Wood J, Maclntyre CR, Kelly H, Lambert SB, Gilbert GL, et al. Sustained measles elimination in Australia and priorities for long term maintenance. Vaccine. 2007;25(18):3574-80. http://dx.doi.org/10.1016/j. vaccine.2007.01.090 PMID:17300858

34. Tischer A, Gassner M, Richard J-L, Suter-Riniker F, Mankertz A, Heininger U. Vaccinated students with negative enzyme immunoassay results show positive measles virus-specific antibody levels by immunofluorescence and plaque neutralisation tests. J Clin Virol. 2007;38(3):204-9. http:// dx.doi.org/10.1016/j.jcv.2006.12.017 PMID:17289430

35. Flipse $W$ and De Schrijver K. Uitbraak van bof onder universiteitsstudenten in 2012 [Mumps outbreak among university students in 2012]. Vlaams Infectieziektebull.
2013;85(3):Article 2. Dutch http://www.infectieziektebulletin. be/defaultSubsite.aspx?id=33294\#.VJ3ImDAA.</unknown〉

36. Agentschap Zorg en Gezondheid, cijfers [Agency for Care and Health]. Brussels: Flemish Governement. Dutch. Available from: http://www.zorg-en-gezondheid.be/cijfers 